\title{
How the intensity of isolated substorms is controlled by the solar wind parameters
}

\author{
Vyacheslav Georgievich Vorobjev ${ }^{1 *}$, Elizaveta Evgenievna Antonova ${ }^{2,3}$ and Oksana Ivanovna Yagodkina ${ }^{1}$
}

\begin{abstract}
Analysis of 163 isolated substorms showed that their intensity quantified as a maximum absolute value of the AL index increases with an increase in the velocity and number density of the solar wind plasma and hence its dynamic pressure. Most of the coupling functions describing the energy loading to the magnetosphere, e.g., the Kan-Lee electric field $\left(E_{\mathrm{KL}}\right)$ and the Newell factor $(\mathrm{d} \Phi / \mathrm{d} t)$, do not include the dynamic pressure as an input parameter. Having examined the correlation between these functions and the dynamic pressure, we found that, surprisingly, while almost uncorrelated for any arbitrary time interval, both $E_{\mathrm{KL}}$ and $\mathrm{d} \Phi / \mathrm{d} t$ correlate with the dynamic pressure within $1 \mathrm{~h}$ before the onset of isolated substorms. That is, an increase in the solar wind dynamic pressure is associated with an increase in the solar wind driving before the onset. We assume that the increase in the dynamic pressure as early as before substorm growth path creates the conditions inside the magnetosphere that impede the occurrence of substorms and increase the threshold for the instability leading to expansion onset, forcing the accumulation of greater amount of energy in the magnetosphere. This energy is released during substorm expansion, producing a more intense magnetic bay.
\end{abstract}

Keywords: Isolated substorms, Substorm onset, The AL index, Solar wind plasma, Solar wind driving

\section{Introduction}

The concept of classical substorm is based on the loading of solar wind energy into the Earth's magnetosphere followed by a sudden release of this energy during substorm expansion. To characterize the time interval of the loading, which typically lasts $0.5-2 \mathrm{~h}$, McPherron (1970) introduced the term "growth phase." It was inferred from the number of observations that the growth phase starts when the $B z$ component of the interplanetary magnetic field (IMF) turns southward. This approach was subsequently supported by many experimental and theoretical works (see, for example, Russell and McPherron 1973; Shukhtina et al. 2005; Boakes et al. 2009).

However, despite extended efforts in the study of magnetospheric substorms, there are many key problems, which remain unsolved, such as a location of substorm onset and the mechanism leading to a sudden release of energy. A relationship between substorm processes and

\footnotetext{
*Correspondence: vorobjev@pgia.ru

1 Polar Geophysical Institute, Apatity, Murmansk Region, Russia Full list of author information is available at the end of the article
}

ring current development is unclear either. The latter problem is especially important taking into account the recent results of Antonova et al. $(2014,2015,2017)$ and Kirpichev et al. (2016), who showed that a significant part of the auroral oval maps to the plasma ring that surrounds the Earth. Accordingly, the transverse currents in this ring are a high-latitude continuation of the ring current.

The dependence of loading-unloading processes on the parameters of the solar wind and IMF is one of the unsolved problems of the magnetospheric substorm physics. In particular, the relationship between intensity of an isolated substorm (characterized by the maximum absolute value of the AL index) and these parameters is unknown. Results of previous studies are ambiguous and often contradictory. For example, Kallio et al. (2000) concluded that substorm intensity is determined by the direct input of energy during the expansive phase, which contradicts the results of Lyons (1996) and Lyons et al. (1997), who found that a number of substorm onsets are associated with a northward turning of the IMF $B z$ component. Shukhtina et al. (2005) and Milan et al. (2009) 
showed that the intensity of substorms is determined by the total magnetic flux transported from the dayside magnetosphere to the tail before substorm onset. However, later $\mathrm{Li}$ et al. (2013) demonstrated that the intensity of substorms correlates well with the electric field determined by the Kan-Lee formula (Kan and Lee 1979), which is independent of the total amount of energy uploaded to the magnetosphere during the growth phase. Vorobjev et al. (2016) made a statistical study of the loading-unloading processes of the magnetosphere during isolated substorms and showed that there is a strong relationship between the integral values of energy uploaded to the magnetosphere during the growth and expansive phases and the substorm intensity quantified as a total energy of auroral precipitation.

Therefore, to establish the factor controlling substorm intensity, which varies in a wide range, is a fundamental unsolved problem. It is clear that the solar wind plasma and the IMF are the main sources of the magnetospheric substorm energy. Conventionally, it is assumed that the $\mathrm{Bz}$ component of the IMF is responsible for substorm generation. The solar wind velocity $(V)$ and number density $(N)$, and hence the dynamic pressure $(P)$, do not change significantly on the timescales of substorm and usually are not considered as independent sources of substorms. However, there are some studies, which point to a possible importance of the solar wind kinetic energy for substorm development. For example, Barkhatov et al. (2017) showed that the prediction of the AL index variations using the neural networks is more successful when the input parameters contain not only the IMF components, but also the dynamic pressure integrated over 2-3 h before substorm onset. At the same time, Newell et al. $(2013,2016)$ studied the long-term effects of substorms, including diurnal, seasonal, and annual variations, and showed that the probability to predict the appearance of substorm series increases when the solar wind velocity is used as a unique input parameter instead of more complicated functions, which include the components of the IMF. The effect of the dynamic pressure is not significant. The results by Maggiolo et al. (2017) suggest that the solar wind velocity's dominant impact on geomagnetic activity is caused by the compression regions at the interface of fast/slow solar wind regimes, which are very geo-effective, as they are associated with high solar wind pressure and strong interplanetary magnetic field.

In the present study, we test the hypothesis that prior to the substorm growth phase the solar wind plasma parameters (generally $\mathrm{N}$ and $\mathrm{Pd}$ ) not only determine the magnetospheric state, but also to a large extent determine the intensity of a following isolated substorm. The characteristics of this magnetospheric state defined by solar wind $V, N$ and $P$ are responsible for the amount of energy uploaded during the growth phase and subsequently released during the expansive phase, and hence for the intensity of a substorm. Therefore, we study the behavior of the IMF components, as well as of the solar wind velocity, number density, and dynamic pressure, preceding isolated substorms of different intensities, in order to examine their influence on the energy loading to the magnetosphere before substorm onset. The dependence of the loading on the SYM/H index of geomagnetic activity is also investigated.

\section{Data analysis}

In the current study, we used the list of 163 isolated substorms of different intensities available at http://pgia.ru/ lang/en/data. The isolated substorms included in this list were selected using diurnal variations and 1-min values of the AL index during the winter seasons from 1995 to 2013, according to the following criteria:

1. The time interval between two consecutive substorms should be at least $3 \mathrm{~h}$.

2. The AL magnitude should not exceed $1500 \mathrm{nT}$.

3. The substorm duration should not be less than $3 \mathrm{~h}$.

4. The time of the end of substorm corresponds to the instant at which the absolute value of $\mathrm{AL}$ is $<0.2$ of the absolute value of AL minimum.

The selection of substorms in accordance with criteria 1-3 was performed by visual inspection of the AL index diurnal variations, while the time of onset $\left(T_{0}\right)$ corresponding to the $n$th $\mathrm{AL}$ index was determined as follows. For substorms with minimum $\mathrm{AL}$ less than $-300 \mathrm{nT}, T_{0}$ corresponded to the time for which $\left|\left(\mathrm{AL}_{n}-\mathrm{AL}_{n-1}\right)\right|>60 \mathrm{nT}$ and $\left|\left(\mathrm{AL}_{n+1}-\mathrm{AL}_{n-1}\right)\right|>100 \mathrm{nT}$. For weak isolated substorms $\left(\mathrm{AL}_{\min }>-300 \mathrm{nT}\right)$ a simpler criterion was used: $\left|\left(\mathrm{AL}_{n}-\mathrm{AL}_{\mathrm{n}-1}\right)\right|>30 \mathrm{nT}$. (Please see Vorobjev et al. (2016) for details.)

The solar wind parameters, IMF, and geomagnetic indices were taken from 1-min OMNI database provided by the NASA's Space Physics Data Facility. For each substorm an 8-h interval ( $4 \mathrm{~h}$ before and $4 \mathrm{~h}$ after $T_{0}$ ) was inspected to eliminate the events for which data gaps of more than 10-min duration were present in the time series.

\section{Generation conditions for substorms of different intensities}

All selected substorms were divided into three groups, depending on the minimum value of $\mathrm{AL}$ variation: weak $\left(\mathrm{AL}_{\min }>-300 \mathrm{nT}\right)$, moderate $\left(-600 \mathrm{nT}<\mathrm{AL}_{\min }<-300 \mathrm{nT}\right), \quad$ and strong $\left(-1500 \mathrm{nT}<\mathrm{AL}_{\min }<-600 \mathrm{nT}\right)$. For each group we 
calculated the average values of $\mathrm{AL}_{\text {min }}$, as well as the values of the solar wind parameters and PC and SYM/H indices averaged over 1-h interval that started 1,2 and $3 \mathrm{~h}$ before onset. Figure 1 shows the results of averaging over one (the black circles, bold line), two (the pluses, solid line), and three (the crosses, dashed line) hours before $T_{0}$. As can be seen, the three curves are very close to each other, especially for solar wind velocity and number density. This means that the solar wind $V, N$, and hence $P$ before an isolated substorm are very stable during at least $3 \mathrm{~h}$ (see Fig. 1a, b), increasing with $\left|\left\langle\mathrm{AL}_{\text {min }}\right\rangle\right|$ increasing. Figure $1 c, d$ shows that the SYM/H index decreases and the PC index increases with $\left|\left\langle\mathrm{AL}_{\text {min }}\right\rangle\right|$ increasing, and the geomagnetic activity $1 \mathrm{~h}$ before $T_{0}$ [typical timescale of the growth phase, according to McPherron (1970)] tends to be higher.

It is important to note that higher values of $V, N, \mathrm{PC}$, and $|\mathrm{SYM} / \mathrm{H}|$ lead to stronger isolated substorms. This tendency holds for all time intervals, even for that of $3 \mathrm{~h}$ before $T_{0}$. For weak substorms the average velocity does not exceed $400 \mathrm{~km} / \mathrm{s}$, while for strong substorms it is higher than $450 \mathrm{~km} / \mathrm{s}$. The average number density is $\sim 6 \mathrm{~cm}^{-3}$ and $\sim 9 \mathrm{~cm}^{-3}$ for weak and strong substorms, respectively. The weak substorms take place when $\mathrm{SYM} / \mathrm{H} \sim-5 \mathrm{nT}$, while the strong ones occur when $\mathrm{SYM} / \mathrm{H} \sim-16 \mathrm{nT}$.

While an increase in the geomagnetic indices before strong substorms (see Fig. 1c, d) can be anticipated, a simultaneous increase in both solar wind velocity and density (and hence the dynamic pressure) is unexpected, taking into account that these two quantities tend to be anticorrelated: The high-speed flows of the solar wind that originated in the coronal holes have low density, while the dense solar wind streams in the region of the heliospheric current sheet have relatively low speeds. Figure $2 \mathrm{a}, \mathrm{b}$ shows a relation between the values of the solar wind plasma density and velocity for all analyzed events averaged over $1 \mathrm{~h}$ before $T_{0}$ (Fig. $2 \mathrm{a}$, the red dots) and after $T_{0}$ (Fig. 2b, the blue dots). The straight lines correspond to the best linear fit. As seen from Fig. 2a,
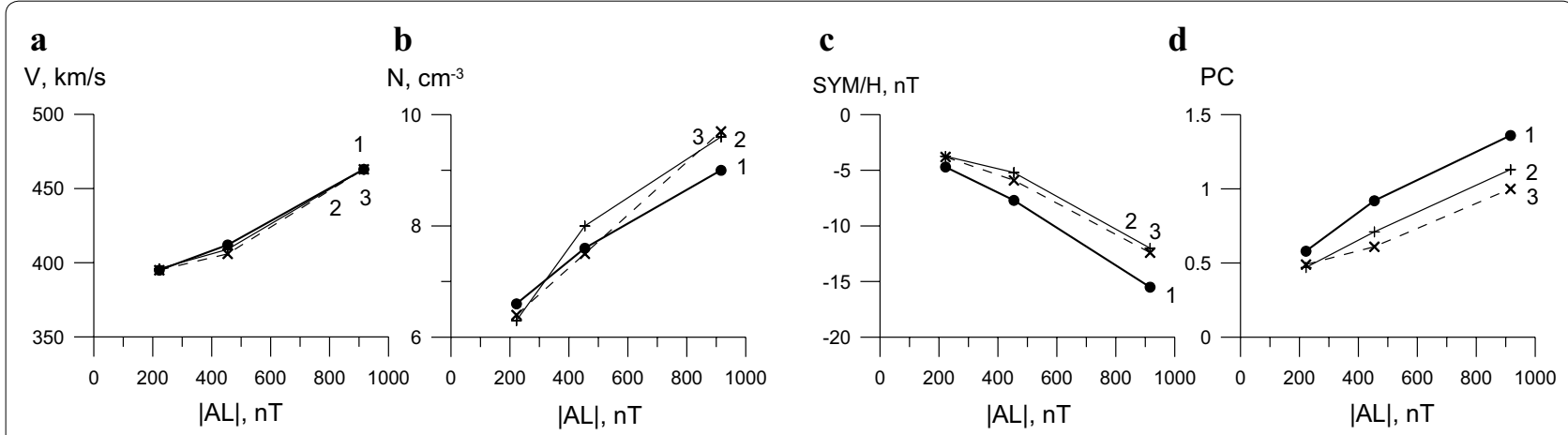

Fig. 1 Average values of the solar wind velocity (a) and number density (b); SYM/H (c) and PC (d) indices of geomagnetic activity versus substorm intensity. The averaging is performed over 1-h interval starting one (curve 1), two (curve 2), and three (curve 3 ) hours before substorm onset
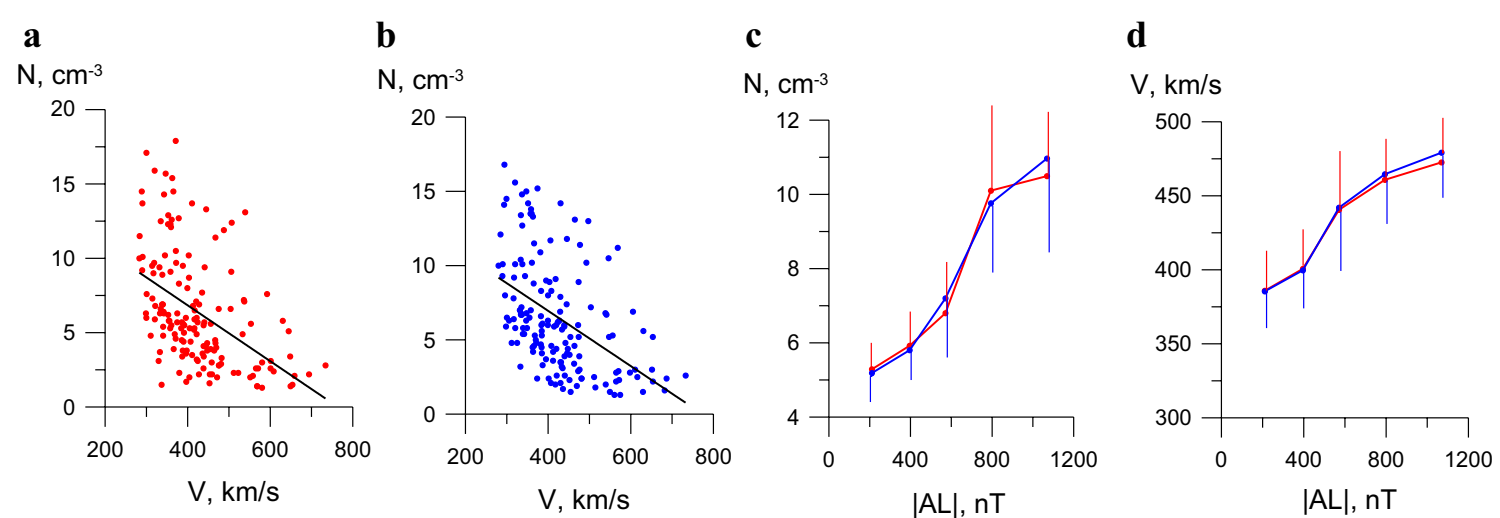

Fig. 2 Relationship between the solar wind plasma density and velocity for the time intervals of $1 \mathrm{~h}$ before (a red dots) and after (b blue dots) substorm onset. The average values of the solar wind density (c) and velocity (d) for substorms of different intensities. The averaging is made $1 \mathrm{~h}$ before (red) and $1 \mathrm{~h}$ after (blue) onsets. Data are averaged in the 200-nT bins starting from $100 \mathrm{nT}$ 
b, the number density tends to decrease with velocity increasing. However, both $N$ and $V$ show an increase with increasing intensity of isolated substorms (see Fig. 2c, d).

In summary, it was found that the solar wind velocity and number density are almost constant during a long time interval covering at least $3 \mathrm{~h}$ before and $1 \mathrm{~h}$ after the onset. However, the minimum value of AL variation decreases with increasing average values of the velocity and density, and hence of the dynamic pressure. We interpret this effect as follows. The steady state of the magnetosphere before an isolated substorm depends on the parameters of the solar wind. The higher values of $N$ and $V$ create the conditions that constrain substorm development. In other words, for higher dynamic pressure it is necessary to load larger amount of energy into the magnetosphere during the growth phase to be able to achieve the threshold, which should be overcome for substorm generation. Subsequently, this energy releases during the expansion phase, leading to the stronger substorms.

\section{How the solar wind density, velocity, and pressure are related to some coupling functions containing the IMF values}

Recently, Vorobjev et al.(2016) have showed that the intensity of isolated substorms strongly correlates with the Kan and Lee (1979) electric field $E_{\mathrm{KL}}=V \cdot B_{T} \cdot \sin ^{2}(/ 2)$, and the Newell et al. (2007) parameter $\mathrm{d} \Phi / \mathrm{d} t=V^{4 / 3} \cdot B_{T}^{3 / 3}$ $\cdot \sin ^{8 / 3}(/ 2)\left(\right.$ where $B_{T}=\left(B y^{2}+B z^{2}\right)^{1 / 2}$, and $=\operatorname{arctg}(B y /$ $B z)$ ). Therefore, it is important to clarify whether there is a correlation between these functions and the solar wind $V, N$, and $P$. We also included in the analysis the sign of the IMF $B z$ component, considering that according to many authors the behavior of $B z$ component is very important for substorm development.

Figure 3 shows the relation between the solar wind velocity (Fig. 3a), number density (Fig. 3b), and dynamic
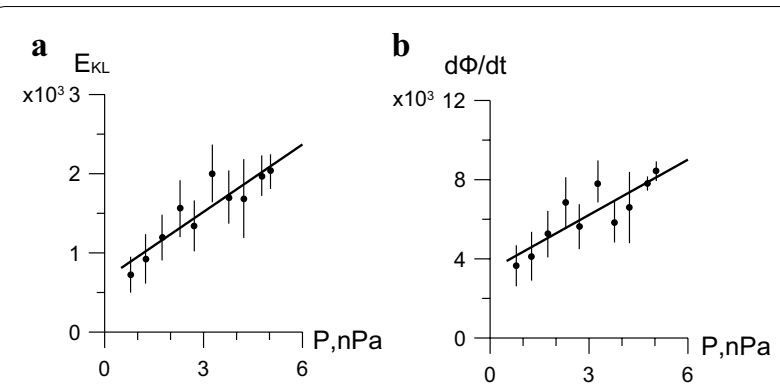

Fig. 4 Values of $E_{K L}$ and $d \Phi / d t$, which characterize the solar wind driving before the substorm expansive phase versus solar wind dynamic pressure. The dots represent the average values of the functions binned every $0.5 \mathrm{nPa}$, the vertical lines indicate the mean square deviation, and the solid lines represent the best linear fits

pressure (Fig. 3c), and the $B z$ component. We used the data $1 \mathrm{~h}$ before substorm onset, averaged for different bins of $B z$ values, taken with a step $2 \mathrm{nT}$. The solid lines represent the best linear fits obtained by the leastsquares method. The correlation coefficients between $V$, $N, P$, and $B z$ for the binned data (in parentheses for the original arrays) are $r=0.31(0.40) ; 0.79(0.55)$ and 0.88 (0.59), correspondingly. The use of the orthogonal regression method does not cause a noticeable modification in the linear fits and correlation coefficients. As can be seen, nearly the same solar wind velocity is observed when $B z$ changes both value and orientation. In fact, Fig. 3a shows the average value of $V$. On the contrary, the values of $N$ and $P$ increase with southern IMF increasing. This result indicates that before isolated substorm onset the loading of the magnetosphere, determined by the level of southern $B z$, increases with the solar wind number density and dynamic pressure.

The solar wind velocity $V$, being one of the components of the coupling functions $E_{\mathrm{KL}}$ and $\mathrm{d} \Phi / \mathrm{d} t$, is also significant. Figure 4 shows the variations of $E_{\mathrm{KL}}$ and $\mathrm{d} \Phi / \mathrm{d} t$ with the dynamic pressure. The mean values of the functions
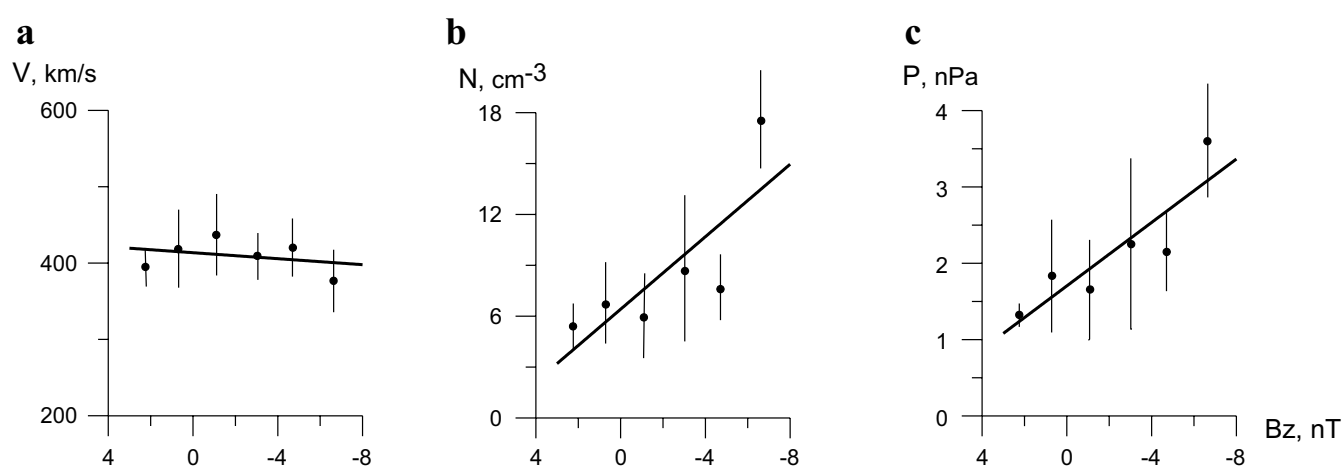

Fig. 3 Relationship between the solar wind velocity (a), plasma density (b) and dynamic pressure (c), and the IMF Bz component. The averaging is performed $1 \mathrm{~h}$ before the substorm onset. The vertical lines indicate the mean square deviation, and the solid lines represent the best linear fits 
obtained by averaging over $1 \mathrm{~h}$ before substorm onset were calculated for 10 bins of the dynamic pressure, with the width of a bin taken as $0.5 \mathrm{nPa}$. As seen from the figure, both $E_{\mathrm{KL}}$ and $\mathrm{d} \Phi / \mathrm{d} t$ strongly correlate with the dynamic pressure and the correlation coefficients are equal to $r=0.81(0.61)$ and $r=0.85(0.58)$, respectively. This means that an increase in the dynamic pressure strengthens the solar wind driving before isolated substorm onset. The original correlation coefficients for basic variables $(N, P, E k l, \mathrm{~d} F / \mathrm{d} t)$ are at level from 0.55 to 0.61 . They are not as exciting as for the binning data, but are rather high to take these correlations into consideration.

There is no reason to assume that such a close relationship between the dynamic pressure and the $E_{\mathrm{KL}}$ and $\mathrm{d} \Phi / \mathrm{d} t$ functions keeps for other randomly selected periods. To support this point of view we selected three arbitrary time intervals in December 2000, 2004 and 2007. December is in the middle of the winter season, and the years 2000 and 2007 correspond to the minimum and maximum of solar activity, respectively, and 2004 is in between. The correlation coefficients for the original arrays are $r=0.12$ and $r=0.09$ for the year 2000, $r=0.07$ and $r=0.06$ for the year 2004, and $r=0.32$ and $r=0.23$ for the year 2007, for $E_{\mathrm{KL}}$ and $\mathrm{d} \Phi / \mathrm{d} t$, respectively.

\section{Discussion and conclusions}

Our results can be analyzed in the framework of the substorm model based on the solar wind energy loading into and the subsequent unloading in the magnetosphere. In this approach, the substorm starts due to development of an instability, which does not require any external trigger to start. To explain the observed effects, it is necessary to analyze the stability of plasma and magnetic field distributions in the magnetosphere under changing conditions in the solar wind and IMF. It is a complex problem, which is far from its final solution. We can only propose some qualitative explanation.

Isolated substorms develop during the time intervals when the Earth's magnetosphere is in quasi-stable states. Each state has a long duration ( $>3 \mathrm{~h}$ ) and corresponds to a specific condition in the solar wind and IMF. Prior to growth phase onset, the interplanetary magnetic field is predominantly northward. In this case, both the transverse currents in the magnetotail and the ring current are weak, and the geomagnetic field is nearly dipolar not only in the inner magnetosphere, but also at considerable distances from the Earth.

The magnetosphere compression by an increased $P$ does not produce the conditions to generate an isolated substorm. A change in the IMF $B z$ component sign leads to a change in transverse and field-aligned current in the magnetosphere and its configuration (see, for example,
Sibeck et al. (1991) and later works). Redistribution of the magnetic flux between dayside and nightside regions of the magnetosphere takes place, which is commonly attributed to the "loading" of the magnetosphere. If the solar wind parameters remain almost unchanged during a few hours, the development of the substorm instability is suppressed by some factors and an increase in the energy uploading before the onset $T_{0}$ is required for substorm generation. Indeed, an increase in the solar wind dynamic pressure leads to an increase in the pressure in the plasma sheet (Tsyganenko and Mukai 2003) and the ring current (see Antonova et al. (2017) and references therein). This permits to assume that the growth of ring current intensity could also play a role of stabilizing factor for substorm instability development. It is known that the existence of vertical magnetic component is unfavorable for the development of tearing (Nishida 1978) and current disruption (Lui et al. 1992) instabilities. According to Antonova et al. $(2013,2017)$, the ring current makes the main contribution into the $\mathrm{SYM} / \mathrm{H}$ variation. This is supported by numerous results including the latest results of Gkioulidou et al. (2014). The compression of the magnetosphere leads to the increase in the ring current, decrease in the magnetic field inside the current, and hence the increase in $|\mathrm{SYM} / \mathrm{H}|$. Simultaneously, the vertical component of the magnetic field increases outside the ring current and near-Earth magnetotail, which increases the thresholds of instabilities such as tearing (mode) and current disruption. Therefore, more energy is required to be uploaded to the magnetosphere during the growth phase to generate substorm. Subsequently, more intense isolated substorms will develop for stronger $|\mathrm{SYM} / \mathrm{H}|$, as shown in Fig. 1c.

Intensity of substorms developed due to instabilities of magnetosphere-ionosphere interactions of field-aligned currents as considered in (Kan et al. 1988; Stepanova et al. 2002) also depends on the solar wing parameters. It should be noted that in the case of $B z>0$, when the solar wind density (and hence the dynamic pressure) increases, plasma in the plasma sheet is getting more dense and cold (Terasawa et al. 1997). Borovsky et al. (1998) showed that the solar wind material reaches the near-Earth nightside plasma sheet in about $4 \mathrm{~h}$, which enhances particle precipitating fluxes and diminishes the average energy of precipitating ions and electrons. In general, when the number density of the solar wind increases, the energy flux of precipitating electrons and hence auroral luminosity in emission $\left(1 \mathrm{NG} \mathrm{N}_{2}{ }^{+}\right)$of $391.4 \mathrm{~nm}$ are reduced (Vorobjev et al. 2004). Consequently, the electron density and conductivity in the $\mathrm{E}$ layer of the ionosphere are also reduced, as well as the intensity of ionospheric and fieldaligned currents, and auroral arcs gradually vanish. This effect leads to stabilization of the instabilities discussed 
by Kan et al. (1988) and Stepanova et al. (2002), which relate substorm onset to the intensification of fieldaligned currents.

All aforementioned factors restrain the generation of substorms, regardless of whether the substorm is generated by instability in the magnetosphere or by an electrostatic instability, developing in the region of upward field-aligned currents (Stepanova et al. 2002; Antonova et al. 2009). Thus, an increase in the solar wind dynamic pressure restrains generation of an isolated substorm.

We cannot exclude a possible alternative explanation of our results that BT as well as its Bx component is effective for substorm generation. Recently, Maggiolo et al. (2017) have reported a significant correlation between $\mathrm{BT}$ and the solar wind dynamic pressure (P). For our purpose, this result implies that if $B z$ correlates with BT, then Bz, EKL and $\mathrm{d} \Phi / \mathrm{d} t$ should correlate with both BT and $P$, providing a possible explanation of correlations presented in Figs. 3 and 4. However, since the IMF vector lies generally in the equatorial plane, one should not expect any correlation between $\mathrm{BT}$ and $\mathrm{Bz}$, and indeed, Maggiolo et al. (2017) report an extremely low level of correlation between BT and $B z$ (their Fig. 4).

In our Fig. 3, $B z$ varies from $-4 \mathrm{nT}$ to $+8 \mathrm{nT}$. We checked the relationship between $\mathrm{BT}$ and $B z$ using monthly intervals of December 2000, 2004 and 2007. As in Fig. 3, all data were fitted by linear regressions with correlation coefficients for bin averages and original arrays being $\sim 0.8$ and $0.1-0.2$, respectively. Despite the large correlation coefficients for bin averages, the slopes of the fitted lines for all three December intervals are very low: the level of BT is $\sim 6 \mathrm{nT}$ in 2000 , varies from 5 to $7 \mathrm{nT}$ in 2004, and varies from 4 to $5 \mathrm{nT}$ in 2007. There is no increase in BT with increasing $B z$ in the selected intervals. This evidence suggests that taking BT into account is not effective to explain the correlations between $P$ and coupling functions EKL and $\mathrm{d} \Phi / \mathrm{d} t$ presented in Fig. 4. Some correlation between $\mathrm{BT}$ and $\mathrm{Bz}$ is possible in our dataset prior to substorm onset when significant correlation between $P$ and $B z$ was discovered (Fig. 3c). As expected, BT increases from $\sim 3$ to $9 \mathrm{nT}$ as $B z$ decreases from 4 to $-8 \mathrm{nT}$, with correlation coefficients for bin averages and original arrays being 0.82 and 0.47 , respectively. Correlation of $\mathrm{BT}$ and $\mathrm{Bz}$ is possible for moderate and strong solar wind driving, when strong BT correlates with strong $N$ and $P$ in the solar wind during solar mass injections and magnetic storms, which are not discussed in this paper which examines only intervals of isolated substorms when solar wind dynamic pressure does not change significantly during long time intervals $(>3 \mathrm{~h}$ ) before onset. The IMF $B z$ component tends to change from northward before the growth phase to southward prior to the substorm, indicating no correlation during such intervals in the events we studied. There is also no correlation between $B z$ and $P$ before substorm growth phase, when the $B z$ is predominantly northward. Yet, the levels of solar wind $N$ and $P$ before growth phase are very significant factors controlling the isolated substorm intensity.

Finally, we conclude that an increase in the solar wind dynamic pressure as early as before substorm growth path results in the formation of the magnetospheric conditions that impede the isolated substorm generation. Therefore, more energy is required to be uploaded into the magnetosphere to generate a substorm. Subsequently, this energy will be released during the substorm expansion phase, generating more intense magnetic bays. We still do not exclude the possibility of alternative explanation of our results; however, at present our concept seems to be the most realistic.

\section{Abbreviation}

IMF: the interplanetary magnetic field.

\section{Authors' contributions \\ All authors offered the same contribution to this study. All authors read and approved the final manuscript.}

\section{Authors' information}

The authors have been in close collaboration between their two organizations for many years.

\section{Author details}

${ }^{1}$ Polar Geophysical Institute, Apatity, Murmansk Region, Russia. ${ }^{2}$ Skobeltsyn Institute of Nuclear Physics, Lomonosov Moscow State University, Moscow, Russia. ${ }^{3}$ Space Research Institute, Moscow, Russia.

\section{Acknowledgements}

We acknowledge the Goddard Space Flight Center, Space Data Facility for the use of the solar wind and IMF data available for free in the site http://cdawe b.gsfc.nasa.gov/. We also acknowledge the Kyoto World Data Center for the use of $\mathrm{AL}$ and SYM/H indices available for free in the site http://wdc.kugi.kyoto -u.ac.jp/, and Oleg Troshichev, Rico Behlke, and Anna NaemiWiller for providing the PC index available for free in the site http://pcindex.org.

\section{Competing interests}

The authors declare that they have no competing interests.

Availability of data and materials

http://pgia.ru/lang/en/data, http://cdaweb.gsfc.nasa.gov/, http://wdc.kugi. kyoto-u.ac.jp/, http://pcindex.org.

Funding

Not applicable.

\section{Publisher's Note}

Springer Nature remains neutral with regard to jurisdictional claims in published maps and institutional affiliations.

Received: 5 March 2018 Accepted: 12 September 2018

Published online: 19 September 2018 


\section{References}

Antonova EE, Kornilov IA, Kornilova TA, Kornilov OI, Stepanova MV (2009) Features of auroral breakup obtained using data of ground-based television observations: case study. Ann Geophys 27:1413-1422. https://doi. org/10.5194/angeo-27-1413-2009

Antonova EE, Kirpichev IP, Vovchenko WV, Stepanova MV, Riazantseva MO, Pulinets MS, Ovchinnikov IL, Znatkova SS (2013) Characteristics of plasma ring, surrounding the Earth at geocentric distances $\sim 7-10 \mathrm{Re}$, and magnetospheric current systems. J Atmos Solar-Terr Phys 99:85-91. https ://doi.org/10.1016/j.jastp.2012.08.013

Antonova EE, Vorobjev VG, Kirpichev IP, Yagodkina OI (2014) Comparison of the plasma pressure distributions over the equatorial plane and at low altitudes under magnetically quiet conditions. Geomag Aeron 54:278-281. https://doi.org/10.1134/S0016793214030025

Antonova EE, Vorobjev VG, Kirpichev IP, Stepanova MV (2015) Problems with mapping the auroral oval and magnetospheric substorms. Earth Planets Space 67:166. https://doi.org/10.1186/s40623-015-0336-6

Antonova EE, Stepanova M, Kirpichev IP, Ovchinnikov IL, Vorobjev VG, Yagodkina OI, Riazanseva MO, Vovchenko W, Pulinets MS, Znatkova SS, Sotnikov NV (2017) Structure of magnetospheric current systems and mapping of high latitude magnetospheric regions to the ionosphere. J Atm Solar-Terr Phys. https://doi.org/10.1016/j.jastp.2017.10.013

Barkhatov NA, Vorobjev VG, Revunov SE, Yagodkina OI (2017) Effect of solar dynamics parameters on the formation of substorm activity. Geomag Aeron 57(3):251-256. https://doi.org/10.1134/S0016793217030021

Boakes PD, Milan SE, Abel GA, Freeman MP, Chisham G, Hubert B (2009) A statistical study of the open magnetic flux content of the magnetosphere at the time of substorm onset. Geophys Res Lett 36:L04105. https://doi. org/10.1029/2008GL037059

Borovsky JE, Thomsen MF, Elphic RC (1998) The driving of the plasma sheet by the solar wind. J Geophys Res 103(A8):17617-17639. https://doi. org/10.1029/97JA02986

Gkioulidou M, Ukhorskiy AY, Mitchell DG, Sotirelis T, Mauk BH, Lanzerotti LJ (2014) The role of small-scale ion injections in the buildup of Earth's ring current pressure: Van Allen Probes observations of the 17 March 2013 storm. J Geophys Res Space Phys 119:7327-7342. https://doi. org/10.1002/2014JA020096

Kallio El, Pulkkinen TI, Koskinen HEJ et al (2000) Loading-unloading processes in the nightside ionosphere. Geophys Res Lett 27(11):1627-1630. https:// doi.org/10.1029/1999GL003694

Kan JR, Lee LC (1979) Energy coupling and the solar wind dynamo. Geophys Res Lett 6(7):577-580. https://doi.org/10.1029/GL006i007p00577

Kan JR, Zhu L, Akasofu S-I (1988) A theory of substorms: onset and subsidence. J Geophys Res 93(A6):5624-5640

Kirpichev IP, Yagodkina OI, Vorobjev VG, Antonova EE (2016) Position of projections of the nightside auroral oval equatorward and poleward edges in the magnetosphere equatorial plane. Geomag Aeron 56(4):407-414. https://doi.org/10.1134/S001679321604006X

Li H, Wang C, Peng Z (2013) Solar wind impact on growth phase duration and substorm intensity. J Geophys Res 118:4270-4278. https://doi. org/10.1002/jgra.50399

Lui ATY, Lopez RE, Anderson BJ et al (1992) Current disruptions in the NearEarth neutral sheet region. J Geophys Res 97(A2):1461-1480. https://doi. org/10.1029/91JA02401

Lyons LR (1996) Substorms: fundamental observational features, distinction from other disturbances, and external triggering. J Geophys Res 101(A6):13011-13026. https://doi.org/10.1029/95JA01987
Lyons LR, Blanchard GT, Samson JC, Lepping RP, Yamamoto T, Moretto T (1997) Coordinated observations demonstrating external substorm triggering. J Geophys Res 102(A12):27039-27051. https://doi.org/10.1029/97JA02639

Maggiolo R, Hamrin M, De Keyser J, Pitkänen T, Cessateur G, Gunell H, Maes $L$ (2017) The delayed time response of geomagnetic activity to the solar wind. J Geophys Res Space Phys 122:11,109-11,127. https://doi. org/10.1002/2016ja023793

McPherron RL (1970) Growth phase of magnetospheric substorms. J Geophys Res 75(28):5592-5599

Milan SE, Grocott A, Forsyth C, Imber SM, Boakes PD, Hubert B (2009) A superposed epoch analysis of auroral evolution during substorm growth, onset and recovery: open magnetic flux control of substorm intensity. Ann Geophys 27(2):659-668. https://doi.org/10.5194/angeo-27-659-20092

Newell PT, Sotirelis T, Liou K, Meng C-I, Rich FJ (2007) A nearly universal solar wind-magnetosphere coupling function inferred from 10 magnetospheric state variables. J Geophys Res 112:A01206. https://doi. org/10.1029/2006JA012015

Newell PT, Gjeroev JW, Mitchell EJ (2013) Space climate implications from substorm frequency. J Geophys Res 118(10):6254-6265. https://doi. org/10.1002/jgra.50597

Newell PT, Liou K, Gjeroev JW, Sotirelis T, Wing S, Mitchell EJ (2016) Substorm probabilities are best predicted from solar wind speed. J Atmos Solar-Terr Phys 146:28-37. https://doi.org/10.1016/j.jastp.2016.04.019

Nishida A (1978) Geomagnetic diagnosis of the magnetosphere. Physics and chemistry in space. Springer, New York

Russell CT, McPherron RL (1973) The magnetotail and substorms. Space Sci Rev 15(2-3):205-266. https://doi.org/10.1007/BF00169321

Shukhtina MA, Dmitrieva NP, Popova NG, Sergeev VA, Yahnin AG, Despirak IV (2005) Observational evidence of the loading-unloading substorm scheme. Geophys Res Lett 32:L17107. https://doi.org/10.1029/2005G L023779

Sibeck DG, Lopez RE, Roelof EC (1991) Solar wind control of the magnetopause shape, location and motion. J Geophys Res 96:5489-5495. https:// doi.org/10.1029/90JA02464

Stepanova MV, Antonova EE, Bosqued JM, Kovrazhkin RA, Aubel KR (2002) Asymmetry of auroral electron precipitations and its relationship to the substorm expansion phase onset. J Geophys Res 107(A7):1134. https:// doi.org/10.1029/2001ja003503

Terasawa T, Fujimoto M, Mukai T et al (1997) Solar wind control of density and temperature in the near - earth plasma sheet WIND/GEOTAIL collaboration. Geophys Res Lett 24(80):935-938. https://doi.org/10.1029/96gl0 4018

Tsyganenko NA, Mukai T (2003) Tail plasma sheet models derived from Geotail particle data. J Geophys Res 108(A3):1136. https://doi.org/10.1029/2002J A009707

Vorobjev VG, Rezhenov BV, Yagodkina OI (2004) The solar wind plasma density control of night-time auroral particle precipitation. Ann Geophys 22:1047-1052. https://doi.org/10.5194/angeo-22-1047-2004

Vorobjev VG, Yagodkina OI, Zverev VL (2016) Investigation of isolated substorms: generation conditions and characteristics of different phases. Geomag Aeron 56(6):682-693. https://doi.org/10.1134/S00167932160601 65 